\title{
Ultrafast electron diffraction of THz-excited nanostructures
}

\author{
Kathrin J. Mohler ${ }^{1,2, *}$, and Peter Baum ${ }^{1,2,3, *}$ \\ ${ }^{1}$ Ludwig-Maximilians-Universität München, Am Coulombwall 1, 85748 Garching, Germany \\ ${ }^{2}$ Max Planck Institute of Quantum Optics, Hans-Kopfermann-Straße 1, 85748 Garching, Germany \\ ${ }^{3}$ Universität Konstanz, Universitätsstraße 10, 78464 Konstanz, Germany
}

\begin{abstract}
We study the electromagnetic response of nanostructures to single-cycle $\mathrm{THz}$ excitation by using ultrafast electron diffraction. Although the nanostructures themselves are static, there exist complex subTHz-cycle Bragg spot dynamics that relate via time-dependent AharonovBohm-like phase shifts to the nanoscale electromagnetic potentials.
\end{abstract}

\section{Metamaterials and ultrafast electron diffraction}

Significant progress towards nanoscale optical devices with novel functionalities is currently being made by manipulating electromagnetic waves on a sub-wavelength scale with so-called metamaterials [1]. As 2D assemblies of building blocks with sub-wavelength dimensions, metasurfaces [2] obtain their unprecedented functionality from the shape and periodic arrangement of the structural elements in combination with the corresponding oscillating nanoscale electromagnetic fields. Thereby, optical effects exceeding the ones achieved with natural materials can be realized which paves the way for novel imaging and sensing applications with flat and lightweight optics. Waveform electron microscopy [3] has been demonstrated to reveal such fields in single resonators. Metamaterials, however, are typically rather arrays than individual elements and time-resolved electron diffraction should be a viable approach for understanding the underlying fundamental electrodynamics.

Here, we demonstrate that electron diffraction with sub-excitation-cycle time resolution can indeed reveal phase maps related to the locally induced electromagnetic potentials in space and time. We first report on a theoretical framework for relating measured time-frozen electron diffraction patterns to nanoscale electromagnetic field distributions, and in a second step support these theoretical findings by some first proof-ofprinciple experimental results of a nanostructure array under single-cycle $\mathrm{THz}$ irradiation.

\section{Concept and experimental pump-probe setup}

For this pilot study, wavy periodic structures serving as nanoscale test samples were fabricated by electron beam lithography. The nanostructures (Fig. 1a, left, in black) block the electrons while the gaps (blue-violet) allow for transmission. For unit cells smaller than the transverse coherence of our electron pulses, the de Broglie waves interfere coherently causing the appearance of a $2 \mathrm{D}$ diffraction pattern (Fig. 1a, right). To study the dynamic

*Corresponding authors: kathrin.mohler@physik.lmu.de, peter.baum@lmu.de 
response of our sample to incident $\mathrm{THz}$ radiation, we invoke pump-probe single-electron diffraction [4]. Single-cycle THz pump pulses initiate collective electronic motion in the nanostructures. The induced electromagnetic fields are probed in a time-resolved manner by ultrashort 70-keV electron pulses. An all-optical approach for electron generation, pulse compression and sample excitation (Fig.1b) provides the required time resolution and synchronization between the different stages of our setup [5]. Time-compressed electron pulses with durations much shorter than the excitation cycle ensure that the electrons capture time-frozen snapshots of the THz-induced electromagnetic field distributions while passing through the sample. The de Broglie waves of electrons passing through an electromagnetic field are subject to phase shifts depending on the electron velocity, the arrival time and the lateral position with respect to the local electromagnetic potentials.

(a)

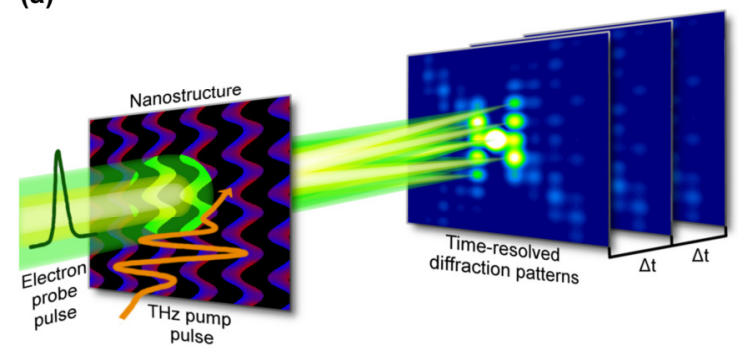

(b)

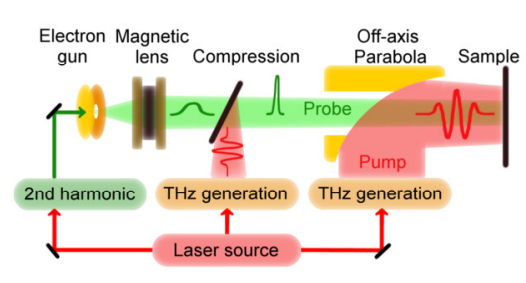

Fig. 1. Concept for sub-cycle nanodiffraction and experimental setup. (a) Optically induced electronic motion in nanostructures is probed via time-frozen electron diffraction with sub-optical-cycle electron pulses. (b) Pump-probe setup for electron diffraction with time-compressed electron pulses.

In diffraction, the phase shifts manifest themselves as intensity changes of individual Bragg spots originating from the sub-unit cell dynamics, typically accompanied by global deflection of the entire pattern. Thus, information about the local field distributions inside the sample at the time of electron transit can be extracted from the diffraction.

\section{Theoretical framework}

First, we theoretically analyze the interaction of electron pulses with dynamic E and B fields, aiming for the prediction and interpretation of the expected diffraction phenomena. The electromagnetic response of our nanostructures to the optical pump pulse is calculated from the finite-difference time-domain solution of Maxwell's equations. The resulting electromagnetic fields are transformed into the corresponding electromagnetic 4-potential. The expected time-dependent phase shifts acquired by the electrons along their trajectories are determined by generalizing the Aharonov-Bohm formulas to non-zero electromagnetic fields $[6,7]$. We see that magnetic and electric contributions can be distinguished by their different velocity dependences [3]. Finally, the merged effect of the static nanostructures and the dynamic electromagnetic fields on the electron diffraction pattern is modelled by combining a stationary absorption mask [8] with the above-mentioned time-dependent phase mask, followed by a Fourier transform. For realistic experimental scenarios, this simulation framework predicts a rich diffraction pattern (as depicted in Fig. 1a), with Bragg spot intensity changes in the percent range following the dynamics of the $\mathrm{THz}$ excitation.

\section{First experimental results}

Second, we report preliminary experimental data. Figure 2a shows a series of time-resolved diffraction patterns acquired for different $\mathrm{THz}$-electron time delays. In order to reveal the 
dynamics, we integrated along the y direction, perpendicular to the incident $\mathrm{THz}$ polarization (Fig. 2b). In accordance with the theoretical framework, we see the singlecycle characteristics of the $\mathrm{THz}$ excitation as a global deflection of the entire electron diffraction pattern, while sub-unit-cell dynamics become evident in the time-dependent relative intensity changes of the individual Bragg spots. Apart from the Bragg spot dynamics of interest, there are also some streaking effects in the current data arising from the tiny deflection angles for $70-\mathrm{keV}$ electrons in combination with Bragg spot broadening due to the limited temporal resolution of $\sim 150 \mathrm{fs}$ for this particular scan.

(a)

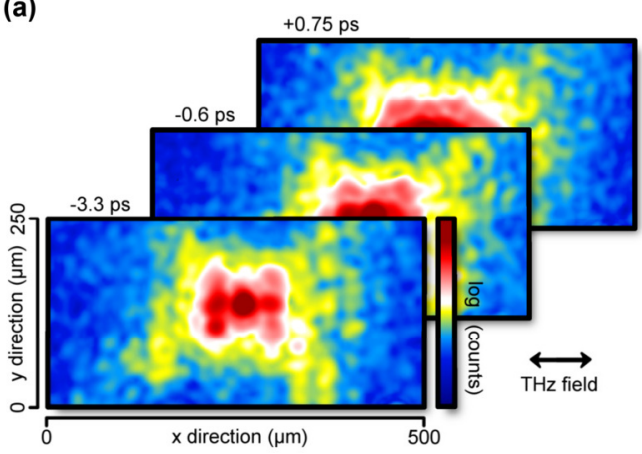

(b)

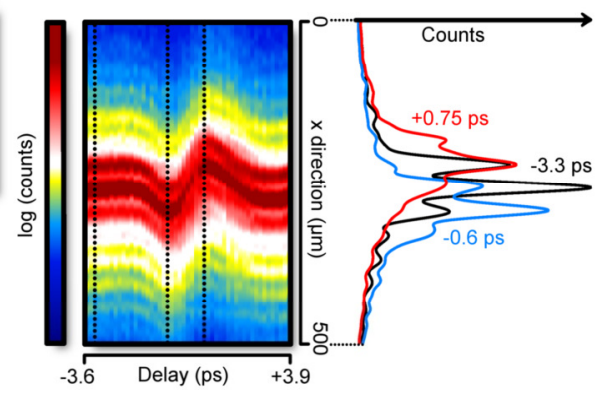

Fig. 2. Experimental time-resolved diffraction. (a) Electron diffraction patterns for different THzelectron delays. (b) Diffraction results integrated along the y direction showing the single-cycle $\mathrm{THz}$ characteristics in the global deflection and delay-dependent Bragg spot intensity changes.

Shorter electron pulses in the tens of femtosecond range $[5,9]$, an electron gun with a better spatial coherence and a more collinear excitation geometry with suppressed global deflection effects should allow for the unobstructed, direct measurement of the electromagnetic dynamics in our nanostructure array and, according to the simulations above, also in much more complicated metamaterials. Reducing the electron pulse durations towards the attosecond regime $[10,11]$ will directly allow for the extension of this method from $\mathrm{THz}$ to optical frequencies, closer to the technologically useful resonances.

We thank Rupert Huber and Imke Gronwald for discussions and sample preparation. This work was supported by the ERC consolidator grant "DIVI" and the Munich centre for Advanced Photonics.

\section{References}

1. W. Withayachumnankul, D. Abbott, IEEE Photon. J. 1(2), 99-118 (2009).

2. N. Yu, F. Capasso, Nat. Mater. 13, 139-150 (2014).

3. A. Ryabov, P. Baum, Science 353, 374-377 (2016).

4. S. Lahme, C. Kealhofer, F. Krausz, P. Baum, Struct. Dyn. 1, 034303 (2014).

5. C. Kealhofer, W. Schneider, D. Ehberger, A. Ryabov, F. Krausz, P. Baum, Science 352, 429-433 (2016).

6. Y. Aharonov, D. Bohm, Phys. Rev. 115, 485-491 (1959).

7. M. Bright, D. Singleton, A. Yoshida, Eur. Phys. J. C 75, 446 (2015).

8. B. McMorran, J. D. Perreault, T. A. Savas, A. Cronin, Ultramicroscopy 106, 356-364 (2006).

9. A. Gliserin, M. Walbran, F. Krausz, P. Baum, Nat. Commun. 6, 8723 (2015).

10. Y. Morimoto, P. Baum, Nat. Phys. 14, 252-256 (2018).

11. K.E. Priebe, C. Rathje, S. V. Yalunin, T. Hohage, A. Feist, S. Schäfer, C. Ropers, Nat. Photonics 11, 793-797 (2017). 